\title{
The relation between star formation rate and accretion rate in LINERs
}

\author{
Qingwen $\mathrm{Wu}^{1,2}$ and Xinwu $\mathrm{Cao}^{1}$ \\ 1. Shanghai Astronomical Observatory, Chinese Academy of Sciences, Shanghai, 200030, \\ China; qwwu@shao.ac.cn, cxw@shao.ac.cn \\ 2. Graduate School of Chinese Academy of Sciences, Beijing, 100039, China
}

\begin{abstract}
It is argued that there is a linear correlation between star formation rate (SFR) and accretion rate for normal bright active galactic nuclei (AGNs). However, it is still unclear whether this correlation holds for LINERs, of which the accretion rates are relatively lower than those of normal bright AGNs. The radiatively inefficient accretion flows (RIAFs) are believed to be present in these LINERs. In this work, we derive accretion rates for a sample of LINERs from their hard X-ray luminosities based on spectral calculations for RIAFs. We find that LINERs follow the same correlation between star formation rate and accretion rate defined by normal bright AGNs, when reasonable parameters are adopted for RIAFs. It means that the gases feed the black hole and star formation in these low-luminosity LINERs may follow the same way as that in normal bright AGNs, which is roughly consistent with recent numerical simulations on quasar evolution.
\end{abstract}

Subject headings: galaxies: active - galaxies: nuclei-X-rays: galaxies - accretion, accretion disks

\section{Introduction}

Black hole $(\mathrm{BH})$ accretion is thought to power AGNs, and the UV/optical bump observed in bright quasars is naturally interpreted as blackbody emission from standard thin accretion disks (e.g., Sun, \& Malkan 1989). The AGN activity may be switched off when the gas near the black hole is exhausted (see Narayan 2002, for a recent review and references therein). Most nearby galactic nuclei are much less active and show very different properties with bright quasars, such as low ionization, lack "UV bump" etc (see Ho 2005, for a review). The accretion mode in these nearby galaxies may be different from that in powerful AGNs. 
Narayan \& Yi (1994) proposed that the standard accretion disk should transit to a RIAF when the accretion rate $\dot{m}\left(=\dot{M} / \dot{M}_{\text {Edd }}\right)$ declines below a critical value $\dot{m}_{\text {crit }}$ within a certain transition radius (e.g., Esin, McClintock, \& Narayan 1997; Yuan \& Narayan 2004; Lu et al. 2004). RIAFs are optically thin, geometrically thick and very hot, which are supposed to be present in many low-luminosity AGNs (e.g., Lasota et al. 1996; Quataert et al. 1999; Gammie et al. 1999; Yuan, et al. 2002; Yuan \& Narayan 2004; Nemmen et al. 2005) and our Galactic center Sgr A* (e.g., Narayan \& Yi 1995).

A very low nuclear-luminosity class of low-ionization nuclear emission-line region (LINER) galaxies were identified by Heckman (1980), in which are found approximately $2 / 3$ low luminosity AGNs (Ho, Filippenko, \& Sargent 1997a). An important fraction of LINERs are clearly weak manifestations of quasar-like phenomena, as demonstrated by the presence of broad $\mathrm{H} \alpha$ emission, which are almost certainly accretion-powered (Ho et al. 1997b). These LINERs can be identified with quiescent $\mathrm{BH}$ remnants from the quasar era. In the present epoch, the supply of the gas available for powering central engines is much curtailed. The low mass accretion rates inferred for many LINERs may suggest that these sources represent the "missing link" between powerful AGNs and normal galaxies as our own.

The recent discovery of the correlation between $\mathrm{BH}$ mass and stellar velocity dispersion in nearby galaxies (e.g., Gebhardt et al. 2000; Ferrarese \& Merritt 2000) demonstrates a fundamental link between the growth of supermassive $\mathrm{BH}$ and bulge formation. The connection between SFR and accretion rate has been explored by some authors (e.g., Heckman et al. 2004; Hao et al. 2005; Satyapal et al. 2005; Dudik et al. 2005). Using the SDSS (Sloan Digital Sky Survey) observations of 123,000 low-redshift galaxies, Heckman et al. (2004) found that the global volume-averaged $\mathrm{SFR} / \dot{M}$ ratio is approximately 1000 in bulge-dominated systems, which is in agreement with the ratio of bulge to $\mathrm{BH}$ mass implied by the $M_{\mathrm{bh}}-\sigma$ relation (Marconi \& Hunt 2003). Hao et al. (2005) also yielded similar results for several ten AGNs (including infrared-selected QSOs, optically-selected QSOs, NLS1s). It was found that the ratio SFR $/ \dot{M}$ of LINERs is apparently different from that of normal bright AGNs, of which the mass accretion rate $\dot{M}$ is estimated by assuming a constant radiative efficiency for all sources. The mass accretion rates $\dot{M}$ derived for LINERs may probably be incorrect (Satyapal et al. 2005), because RIAFs are believed to be in these sources and their radiative efficiencies are lower than these for standard thin disks.

In this paper, we explore whether the LINERs follow the same correlation between accretion rate and SFR defined by normal bright AGNs. The accretion rates are re-estimated for these LINERs based on our spectral calculations for RIAFs. 


\section{The sample}

We adopt the sample given by Satyapal et al. (2005) for our present investigation, which includes 33 LINERs with detected hard X-ray nuclear emission (they are defined as AGN-LINERs in Satyapal et al. (2005)), 54 Seyferts, 15 quasars, 14 radio galaxies, and 14 narrow-line Seyfert 1s (NLS1s). Several LINERs are radio-loud. All sources in this sample have estimated BH masses, FIR and bolometric luminosities. The BH masses of these sources are estimated through resolved stellar kinematics, reverberation mapping, or applying the correlation between optical bulge luminosity and central BH mass derived from nearby galaxies (see Satyapal et al. (2005), for more details). A significant fraction of galaxies in the sample are bulge-dominated and the SFRs of the host galaxies are calculated from $L_{\text {FIR }}$ by using the calibrated formula, in which the old stars' contribution has been properly subtracted (See Eqs. 5 and 6 in Satyapal et al. 2005). The calibrated formula adopted by Satyapal et al. (2005) can also be applied to some early type spiral galaxies in the sample, as this SFR- $L_{\mathrm{FIR}}$ calibration is suggested to be applicable for all type galaxies (Kewley et al. 2002). All data can be found in Satyapal et al. (2005). We note that NGC 3215 is a Wolf-Rayet starburst galaxy, which is excluded in our present investigation. Some typos of their paper are fixed in this paper (see, Satyapal et al. 2006, for erratum).

\section{RIAF spectra}

In order to calculate the spectrum of a RIAF, we need to obtain the RIAF structure. We calculate the global structure of an accretion flow surrounding a Schwarzschild BH using a similar approach as Narayan, Kato, \& Honma (1997). We adopt a simple $\alpha$-viscosity $\left(\tau_{r \phi}=\right.$ $\alpha p$ ) and all radiative processes (Synchrotron, Bremsstrahlung and Compton scattering) are included consistently in our calculations for RIAF structure. The advection by ions and electrons has been considered in the energy equation, and a more realistic state of accreting gas (instead of a polytropic index $\gamma_{\mathrm{g}}$ ) is employed in the calculations, which is similar to that used by Manmoto (2000). The RIAF structure can be calculated when suitable outer

boundary conditions are supplied. After the RIAF structure is obtained, the spectrum of the flow can be calculated in the same way as some previous authors (see, Manmoto 2000; Esin et al. 1996). 


\section{Results}

\subsection{The relation between $L_{X, 2-10 \mathrm{keV}}$ and $\dot{m}$ for RIAF}

Three-dimensional MHD simulations suggest that the viscosity parameter $\alpha$ in the accretion flow is $\sim 0.05-0.2$ (Hawley \& Balbus 2002). Recent observations of black hole binaries (Maccarone 2003) and radio-loud AGNs (Marchesini, Celotti, \& Ferrarese 2004), as well as theoretical accretion models (e.g., Narayan \& Yi 1995; Esin, McClintock, \& Narayan 1997) suggest that the critical accretion rate $\dot{m}_{\text {crit }} \sim 0.01$, below which a RIAF is present. Our numerical calculations show that $\dot{m}_{\text {crit }} \sim 0.01$ requires $\alpha \sim 0.2$, and we adopt $\alpha=0.2$ in all our calculations. The parameter $\beta$ (ratio of gas to total pressure in the accretion flow, $\left.\beta=P_{\mathrm{g}} / P_{\text {tot }}\right)$ is not an independent parameter, which can be related with $\alpha$ as $\beta \approx(6 \alpha-3) /(4 \alpha-3)$, suggested by MHD simulations (Hawley, Gammie, \& Balbus 1996; Narayan et al. 1998). We adopt $\beta \simeq 0.8$ for $\alpha=0.2$. Another parameter in our calculations is the outer radius $R_{\text {out }}$ of the RIAF. We adopt $R_{\text {out }}=200 R_{\mathrm{S}}$ in all our calculations, where the Schwarzschild radius $R_{\mathrm{S}}=2 G M_{\mathrm{bh}} / c^{2}$ and $M_{\mathrm{bh}}$ is the $\mathrm{BH}$ mass. How the outer radius $R_{\text {out }}$ evolves with $\dot{m}$ is still unclear, though it may be a function of $\dot{m}$. We find that the hard $\mathrm{X}$-ray emission is mainly emitted from the inner region of the flow close to the black hole, which is insensitive to the value of $R_{\text {out }}$ adopted. A typical value $\delta=0.1$ (the fraction of the dissipated energy directly heating the electrons) is adopted in all our calculations. Now, we can calculate the structure of the RIAF, and then its spectrum varying with accretion rate $\dot{M}$ when $M_{\mathrm{bh}}$ is specified.

For a fixed BH mass, $M_{\mathrm{bh}}=10^{8} M_{\odot}$, our calculation on the relation between $2-10 \mathrm{keV}$ X-ray luminosity $L_{X, 2-10 \mathrm{keV}}$ and accretion rate (from $\dot{m}=10^{-5}$ up to $\dot{m} \sim \dot{m}_{\text {crit }} \sim 10^{-2}$ ) is plotted in Figure 1. The dependence of $2-10 \mathrm{keV}$ luminosity on $\dot{m}$ can be roughly fitted by single power-law (solid line in Fig. 1),

$$
\log L_{X, 2-10 \mathrm{keV}}=2.37( \pm 0.06) \log \dot{m}+46.33( \pm 0.22),
$$

For $\dot{m} \lesssim 10^{-3.75}, L_{X, 2-10 \mathrm{keV}} \propto \dot{m}^{2}$ and the $2-10 \mathrm{keV}$ X-ray emission is mainly dominated by bremsstrahlung radiation. At higher accretion rate, $10^{-3.75} \lesssim \dot{m} \lesssim 10^{-3.25}$, $L_{X, 2-10 \mathrm{keV}} \propto \dot{m}^{3.5}$ and both the Compton and bremsstrahlung radiation is contributes

to the 2-10 keV X-ray emission. The Comptonized component will dominate the X-ray emission at higher accretion rate $\dot{m} \gtrsim 10^{-3.25}$ which have $L_{X, 2-10 \mathrm{keV}} \propto \dot{m}^{2.2}$ (see dashed line in Fig. 1).

Our spectral calculations show that the $2-10 \mathrm{keV}$ X-ray luminosity is roughly proportional to the $\mathrm{BH}$ mass. So we can calculate the accretion rate using the equation (1), if the $\mathrm{BH}$ mass and $L_{X, 2-10 \mathrm{keV}}$ is given. 


\subsection{The mass accretion rates of LINERs}

The central BH masses for all sources in this sample are available, so we can re-estimate the accretion rates for these 30 low-luminosity LINERs from their hard X-ray luminosity $L_{X, 2-10 \mathrm{keV}}$ based on our RIAF spectral calculations. The remaining three LINERs having $\mathrm{X}$-ray luminosities higher than the maximal values expected by the RIAF model calculation. We estimate the accretion rates of these three sources through $\dot{m}=L_{\text {bol }} / L_{\text {Edd }}$, where $L_{\text {bol }}$ is derived from the X-ray luminosity by using $L_{\mathrm{bol}}=34 L_{X, 2-10 \mathrm{keV}}$. The Eddington ratio of 8 sources in the 14 radio galaxies are lower than $10^{-2}$ and these sources may be also in RIAF state. Four of these 8 sources (3C 31, 3C 84, 3C 338, 3C 465) have intrinsic X-ray luminosity (Evans et al. 2005) and the X-ray emission data for the remaining four sources (3C 88, 3C 285, 3C 327, 3C 402) are not available in the literature. We convert optical core emission measured by Hubble Space Telescope (HST) to a corresponding X-ray luminosity assuming spectral index $\alpha_{o x}=0.6$ for these four sources (Marchesini, Celotti, \& Ferrarese 2004; Falcke, Körding, \& Markoff 2004). Following the same way as LINERs, we re-estimate the accretion rates of these radio galaxies. The re-calculated mass accretion rates and the Satyapal et al. (2005)'s results are listed in Table 1 for comparison.

In Satyapal et al. (2005), they showed that the distributions of SFR/ $\dot{M}$ for LINERs and radio galaxies are apparently different from that of normal bright AGNs. Their estimates on $\dot{M}$ were carried out by assuming a constant radiative efficiency, which may be incorrect for low-luminosity LINERs with RIAFs. We plot the distributions of the ratio of SFR to the re-estimated mass accretion rate $\dot{M}$ for LINERs and radio galaxies in Figure 2 (upper and middle panels). We find that the distributions of SFR/ $\dot{M}$ for LINERs and radio galaxies are similar to that of the normal bright AGNs in this sample(Seyfert, Quasar and NLS1, solid lines in Fig. 2). In Figure 3, we plot the relation of SFR and $\dot{M}$ for the whole sample using our estimates on mass accretion rates $\dot{M}$. The linear regression yields for the normal bright AGNs (Seyfert, Quasar and NLS1):

$$
\log S F R=(0.79 \pm 0.04) \log \dot{M}+(1.66 \pm 0.06)
$$

where SFR and $\dot{M}$ are both in units of $M_{\odot} \mathrm{yr}^{-1}$. The linear regression for the whole sample is:

$$
\log S F R=(0.73 \pm 0.04) \log \dot{M}+(1.55 \pm 0.07) .
$$

We find that the correlations are similar either for the whole sample or the subsample of normal bright AGNs (see, Fig. 3). We have not found any radio galaxies in this sample deviating significantly from the correlation. 


\section{Discussion}

The bolometric luminosities of AGNs can be estimated by integrating their spectral energy distribution or using an empirical relation (e.g., Woo, \& Urry 2002; Elvis et al. 1994; Ho 1999), and then their mass accretion rates are derived if the radiative efficiency is known. A linear correlation between SFR and $\dot{M}$ was found by Satyapal et al. (2005) for normal bright AGNs, while the LINERs in their sample obviously deviate from this correlation, if the same constant radiative efficiency 0.1 is adopted for the whole sample. It was found that the accretion rates for LINERs are significantly lower than those of normal bright AGNs for given SFRs (see Fig. 10 in Satyapal et al. 2005). RIAFs are believed present in these low-luminosity LINERs, and their radiative efficiency $\epsilon_{r}$ is usually much lower than that of standard disks (Narayan \& Yi 1994). This implies the accretion rates of LINERs are underestimated by Satyapal et al. (2005). In this paper, we derive the mass accretion rates of these LINERs from their X-ray luminosities based on RIAF spectral calculations, which should be more reliable.

The standard thin disk is believed present in Seyferts and QSOs and its radiative efficiency $\epsilon_{r}^{0} \sim 0.1$. The slim disk may be present in the NLS1s and its radiative efficiency should be also lower than that of standard disk due to the photon-trapping effects (e.g., Abramowicz et al. 1988; Ohsuga et al. 2002; Chen \& Wang 2004). However, all the Eddington ratios of NLS1s in our sample are less than $6\left(L_{\mathrm{Bol}} / L_{\mathrm{Edd}}\right.$, see table 2 in Satyapal et al. 2005 ) and the radiative efficiencies of slim disks with such Eddington ratios may not deviate much from that of the standard disk (see, dashed line and solid line of Fig. 1 in Ohsuga et al. 2002). So, the derived mass accretion rates should be accurate to a factor of 2 for NLS1s in these sample, which will not alter our main conclusion.

The SFRs is calculated from the FIR luminosity for the whole sample since the FIR luminosity is widely used as a tracer of SFR in galaxies (e.g., Kennicutt 1998; Kewley et al. 2002; Satyapal et al. 2005), though we can not rule out some contribution of dust heating by the AGNs and old stars in elliptical galaxies. However, there are lack of correlations between the Mid-IR and FIR for well-studied PG quasars, which suggests that they are dominated by different heating sources(e.g., Haas et al. 1999). If AGN heating dominates the cooler FIRemitting dust, then there should be a correlation between quasar OUV and FIR luminosity, while such a correlation has not been found(e.g., McMahon, et al. 1999; Isaak et al. 2002; Priddey et al. 2003). In addition, QSOs and Seyferts roughly follow the same universal correlation between the FIR and radio emission deduced from "normal" galaxies, which strongly suggests that FIR emission is still powered by star formation rather by AGNs (e.g., Norris et al. 1988; Luo \& Wu 2005). Although the FIR emission contributed by old stars in some elliptical galaxies could be important (e.g., very few sources in Bell (2003) sample 
have old star contribution, about 4 of 249, higher 80 percent, see Fig. 6 in that paper), the average contribution of the old stars is around 30 percent. In our present SFR calculations, we use the calibrated formula, in which the old star contribution has been subtracted (Bell 2003). So, in statistical sense, the SFRs estimated in our paper are reliable, though we cannot rule out the SFRs of a very few sources in this sample have been overestimated 3-4 times. Even in this case, we believe this has not affected our main statistical results.

We plot our re-estimated accretion rate $\dot{M}$ in Figure 3, and find that all LINERs follow the same correlation defined by the normal bright AGNs (e.g., Seyfert, Quasar and NLS1). The linear correlation between SFR and $\dot{M}$ indicates that black hole accretion evolves in the same way as star formation, which are both regulated by the interstellar gas in the host galaxies. Our results show that the gases feed the black hole and star formation in low luminosity LINERs follow the similar way as luminous normal AGNs. Recent numerical simulations on the quasar activity triggered by the galaxy merger indeed show that the accretion rate $\dot{M}$ and SFR decreases simultaneously with time over several orders of magnitude after the quasar shines at its Eddington limit (Springel et al. 2004; Di Matteo, Springel, \& Hernquist 2005). In their simulations, the gas in the galaxies is driven away by the bright quasar radiation, and then both the star formation and $\mathrm{BH}$ accretion are quenched simultaneously. The SFR is nearly linearly varying with $\dot{M}$ in nearly three orders (e.g., $\dot{M} \sim 5 \times 10^{-4}-5 \times 10^{-1} \mathrm{M}_{\odot} \mathrm{yr}^{-1}$, $\mathrm{SFR} \sim 5 \times 10^{-3}-5 \mathrm{M}_{\odot} \mathrm{yr}^{-1}$ corresponding to $\left.\sim 1.7-2.0 \mathrm{Gyr}\right)$ for the model with galaxies of virial velocity $\mathrm{V}_{\mathrm{vir}}=160 \mathrm{~km} / \mathrm{s}$ (middle red lines in Fig. 2 of Di Matteo, Springel, \& Hernquist 2005). Our results are roughly consistent with their simulations, though the accretion mode transition has not been considered in their simulations.

There are 8 radio galaxies (Eddington ratio less than $10^{-2}$ ) in 14 radio galaxies, of which the accretion rates are derived from their X-ray emission. It is still debating whether the $\mathrm{X}$-ray emission are dominated by the jet emission for some radio galaxies (Falcke, Körding, \& Markoff 2004; Yuan \& Cui 2005). If the jet emission in X-ray bands is important, the accretion rates derived in this paper are only the upper limits, and those 8 triangles in Figure 3 should be shifted towards left direction. As discussed in Sect. 4.2, we have not found these 8 sources deviating from the correlation between SFR and $\dot{M}$, which may imply $\mathrm{X}$-ray emission from the jets is unimportant at least for these 8 sources.

We find that our results are insensitive to any model parameters except $\delta$, which describes the fraction of the viscously dissipated energy directly heating the electrons in the accretion flow. In our calculations, $\delta=0.1$ is adopted, which is a typical value successfully used to model the observed spectra for some low-luminosity AGNs (e.g., Quataert \& Narayan 1999). Although winds may be present in RIAFs, the detailed physics is still unclear and such winds are only described by an artificial power-law parameter $p_{\mathrm{w}}$ (e.g., Blandford \& 
Begelman 1999). In RIAF spectral calculations for X-ray wavebands, the value $\delta$ is somewhat degenerate with $p_{\mathrm{w}}$ (e.g., Quataert \& Narayan 1999). Our calculations show that similar conclusion can be obtained if $\delta=0.3$ and $p_{\mathrm{w}}=0.9$ are adopted for RIAF with winds.

The present sample is a mixture of AGNs, of which both BH masses and bolometric luminosities are estimated. It is difficult to evaluate to what extent the sample is affected

by the selection effects. A more robust sample is desired for testing this relation between $\dot{M}$ and SFR.

The authors are grateful to the referee for constructive suggestions on our paper. We would like to thank Jufu Lu, Feng Yuan, Weimin Gu, Shuangliang Li and Yiqing Lin for helpful discussions on the RIAF model and numerical calculations, and S. Satyapal, and R. P. Dudik, for useful discussions on their data. This work is supported by the National Science Fund for Distinguished Young Scholars (grant 10325314) and the NSFC (grant 10333020).

\section{REFERENCES}

Abramowicz, M. A., et al. 1988, ApJ, 332, 646

Blandford, R. D., \& Begelman, M. C. 1999, MNRAS, 303, L1

Bell, E. F. 2003, ApJ, 586, 794

Chen, L. H., \& Wang, J. M. 2004, ApJ, 604, 101

Di Matteo, T., Springel, V., \& Hernquist, L. 2005, Nature, 433, 604

Dudik, R. P., et al. 2005, ApJ, 620, 113

Elvis, M., et al. 1994, ApJS, 95, 1

Esin, A. A., et al. 1996, ApJ, 465, 312

Esin, A. A., McClintock, J. E, \& Narayan, R. 1997, ApJ, 489, 865

Evans, D. A., et al. 2005, ApJ, in press, astro-ph/0512600

Falcke, H., Körding, E., \& Markoff, S. 2004, A\&A, 414, 895

Ferrarese, L., \& Merritt, D. 2000, ApJ, 539, L9

Gammie, C. F., Narayan, R., \& Blandford, R. 1999, ApJ, 516, 177 
Gebhardt, K., et al. 2000, ApJ, 539, L13

Haas, M., et al. 1999, in The Universe as Seen by ISO, ed. P. Cox \& M. F. Kessler (ESA-SP 427; Noordwijk: ESA), 887

Hao, C. N., et al. 2005, ApJ, 625, 78

Hawley, J. F., Gammie, C. F., \& Balbus, S. A. 1996, ApJ, 464, 690

Hawley, J. F., \& Balbus, S. A. 2002, ApJ, 573, 738

Heckman, T. M. 1980, A\&A, 87, 152

Heckman, T. M., et al. 2004, ApJ, 613, 109

Ho, L. C., Filippenko, A. V., \& Sargent, W. L. W. 1997a, ApJ, 487, 568

Ho, L. C et al. 1997b, ApJS, 112, 391

Ho, L. C. 1999, ApJ, 516, 672

Ho, L. C. 2005, To appear in From X-ray Binaries to Quasars: Black Hole Accretion on All Mass Scales, ed. T. J. Maccarone, R. P. Fender, and L. C. Ho (Dordrecht: Kluwer)(astro-ph/0504643)

Isaak, K. G., et al. 2002, MNRAS, 329, 149

Kennicutt, R. C. 1998, ApJ, 498, 541

Kewley, L. J., et al. 2002, AJ, 124, 3135

Lasota, J. P., et al. 1996, ApJ, 462, 142

Luo, S. G., \& Wu, X. B. 2005, ChJAA, Vol. 5, 448

Lu, J. F., Lin, Y. Q., \& Gu, W. M. 2004, ApJ, 602, 137L

Maccarone, T. J. 2003, A\&A, 409, 697

Manmoto, T. 2000, ApJ, 534, 734

Marchesini, D., Celotti, A., \& Ferrarese, L. 2004, MNRAS, 351, 733

Marconi, A., \& Hunt, L. K. 2003, ApJ, 589, L21

McMahon, R. G., et al. 1999, MNRAS, 309, L1 
Narayan, R., \& Yi, I. 1994, ApJ, 428, L13

Narayan, R., \& Yi, I. 1995, ApJ, 452, 710

Narayan, R., Kato, S., \& Honma, F. 1997, ApJ, 476, 49

Narayan, R., Mahadevan, R., \& Quataert, E. 1998, in Theory of Black Hole Accretion Disks, eds. by M. A. Abramowicz, G. Bjornsson, and J. E. Pringle. (Cambridge University Press), 148

Narayan, R. 2002, in Lighthouses of the Universe, eds. M. Gilfanov, R. Sunyaev, \& E. Churazov (Berlin: Springer), 405

Nemmen, R. S., et al. 2005, ApJ, in press, astro-ph/0512540

Norris, R. P., Allen, D. A., \& Roche, P. F. 1988, MNRAS, 234, 773

Ohsuga, K., et al. 2002, ApJ, 574, 315

Priddey, R. S., et al. 2003, MNRAS, 344, L74

Quataert, E., et al. 1999, ApJ, 525, L89

Quataert, E., \& Narayan, R. 1999, ApJ, 520, 298

Satyapal, S., et al. 2005, ApJ, 633, 86

Satyapal, S., et al. 2006, ApJ, 642, 615

Shakura, N. I., \& Sunyaev, R. A. 1973, A\&A, 24, 337

Springel, V., Di Matteo, T., \& Hernquist, L. 2005, MNRAS, 361, 776

Sun, W. H., \& Malkan, M. A. 1989, ApJ, 346, 68

Woo, J. H., \& Urry, C. M. 2002, ApJ, 579, 530

Yuan, F., et al. 2002, A\&A, 391, 139

Yuan, F., \& Narayan, R. 2004, ApJ, 612, 724

Yuan, F, \& Cui, W. 2005, ApJ, 629, 408 
Table 1. Re-estimated accretion rates of LINERs and Radio galaxies

\begin{tabular}{|c|c|c|c|}
\hline Galaxy Name & $\log _{10} \dot{M}^{\mathrm{a}}$ & $\log _{10} \dot{M} \mathrm{~b}$ & $\log _{10} S F R$ \\
\hline \multicolumn{4}{|l|}{ LINERs $\mathrm{d}$} \\
\hline NGC 4350 & -5.35 & -2.94 & -0.83 \\
\hline NGC 1052 & -2.55 & -1.39 & -0.24 \\
\hline NGC 3031 & -4.05 & -2.33 & -0.76 \\
\hline NGC 4278 & -4.15 & -1.73 & -0.69 \\
\hline NGC 4486 & -3.65 & -1.37 & -0.83 \\
\hline NGC 4579 & -3.25 & -2.04 & 0.09 \\
\hline NGC 6500 & -3.95 & -1.89 & 0.01 \\
\hline NGC 4203 & -4.15 & -2.31 & -0.69 \\
\hline NGC 4494 & -5.35 & -3.15 & -1.22 \\
\hline NGC 4594 & -4.05 & -1.80 & -0.32 \\
\hline NGC 4527 & -5.45 & -2.77 & 0.44 \\
\hline NGC 4125 & -5.55 & -2.61 & -0.62 \\
\hline NGC 4374 & -5.15 & -1.99 & -0.69 \\
\hline NGC 4696 & -4.05 & -2.06 & -0.47 \\
\hline CGCG 162-010 & -2.35 & -0.92 & 1.09 \\
\hline IC 1459 & -3.85 & -1.74 & -0.62 \\
\hline NGC 2787 & -5.95 & -3.39 & -1.16 \\
\hline NGC 315 & -3.00 & -1.14 & 0.01 \\
\hline NGC 2681 & -5.30 & -3.07 & -0.47 \\
\hline NGC 3169 & -4.13 & -2.32 & 0.35 \\
\hline NGC 3245 & -5.06 & -2.56 & -0.16 \\
\hline NGC 3718 & -3.78 & -2.15 & -0.54 \\
\hline NGC 4258 & -4.60 & -2.94 & 0.01 \\
\hline NGC 4261 & -3.71 & -1.73 & -0.39 \\
\hline NGC 4410A & -3.08 & -1.38 & 0.27 \\
\hline NGC 4457 & -5.00 & -3.21 & 0.01 \\
\hline NGC 4552 & -4.67 & -2.33 & -1.03 \\
\hline NGC 4565 & -5.13 & -3.12 & -0.16 \\
\hline NGC 6482 & -3.75 & -1.75 & -0.47 \\
\hline $3 \mathrm{C} 218$ & -2.40 & -1.07 & 0.72 \\
\hline \multicolumn{4}{|c|}{ 3C Radio galaxy d } \\
\hline $3 \mathrm{C} 31$ & -3.751 & -2.12 & 0.35 \\
\hline $3 \mathrm{C} 84$ & -1.751 & -0.53 & 1.18 \\
\hline $3 \mathrm{C} 338$ & -3.451 & -1.64 & 0.35 \\
\hline $3 \mathrm{C} 465$ & -3.151 & -1.21 & 0.35 \\
\hline $3 \mathrm{C} 88$ & -3.351 & -1.41 & 0.44 \\
\hline $3 \mathrm{C} 285$ & -4.251 & -1.79 & 1.15 \\
\hline $3 \mathrm{C} 327$ & -3.351 & -1.10 & 1.54 \\
\hline $3 \mathrm{C} 402$ & -3.351 & -1.53 & 0.44 \\
\hline
\end{tabular}

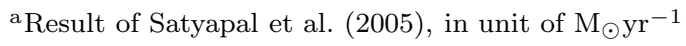

${ }^{\mathrm{b}}$ Our result, in unit of $\mathrm{M}_{\odot} \mathrm{yr}^{-1}$

${ }^{\mathrm{c}}$ in unit of $\mathrm{M}_{\odot} \mathrm{yr}^{-1}$ 
d The accretion rates of remaining 3 LINERs and 6 radio galaxies are estimated from $\dot{m}=L_{\mathrm{bol}} / L_{\mathrm{Edd}}$ with radiative efficiency $\epsilon_{r}^{0}=0.1$. Because the $L_{X, 2-10 \mathrm{keV}}$ of these sources are larger than the maximal values predicted by RIAF model with parameters adopted in our paper and RIAFs is assumed not present in these sources. 


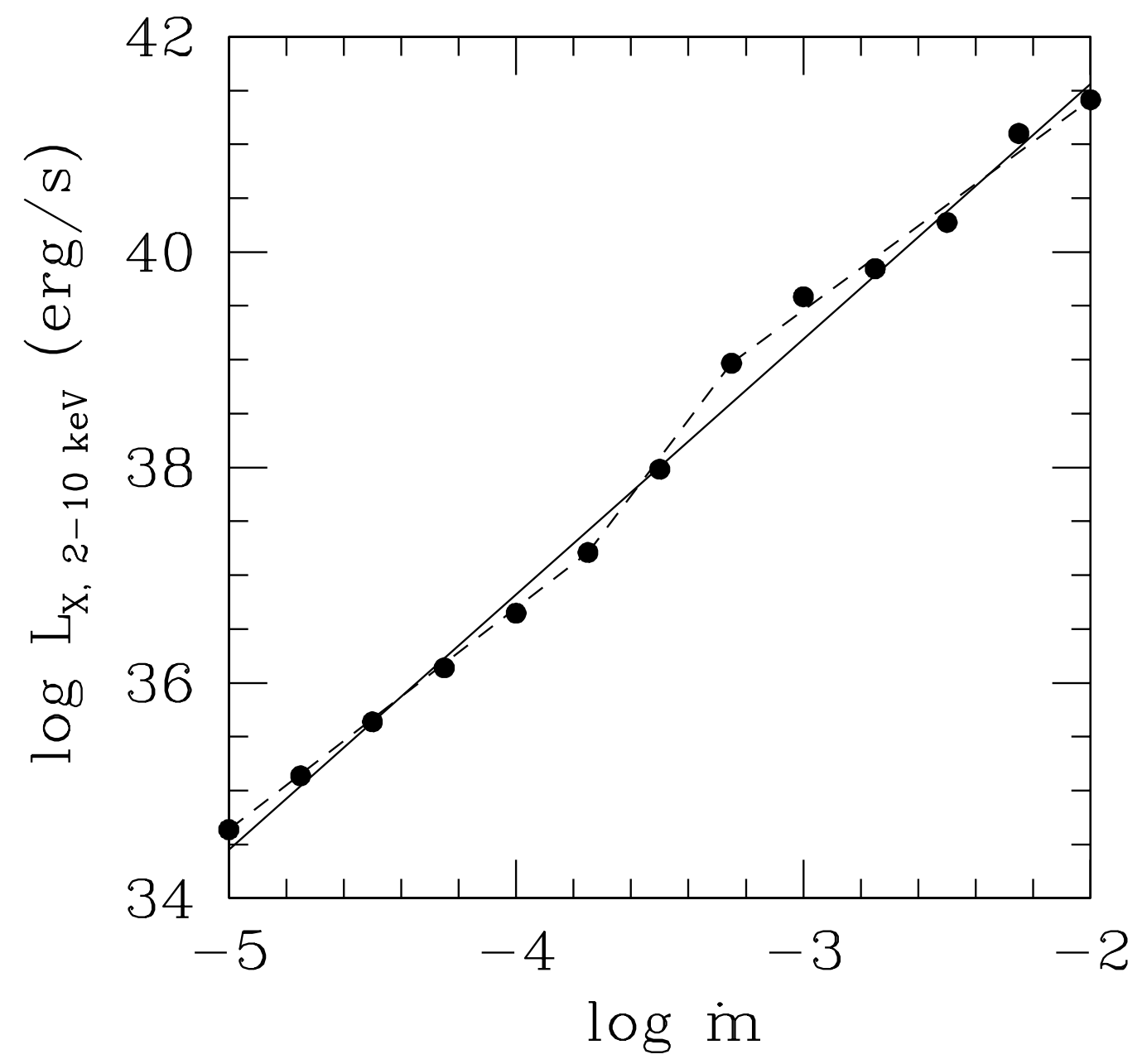

Fig. 1. - The filled circles connected by the dashed line show the integrated 2-10 keV X-ray luminosity versus the dimensionless accretion rate $\dot{m}$ predicted by the RIAF model. The solid line is a linear fit of $L_{X, 2-10 \mathrm{keV}}$ to the model calculated points (filled circles). Model calculations are carried out for a $10^{8} M_{\odot}$ black hole, with $\alpha=0.2, \beta=0.8$ and $\delta=0.1$. 


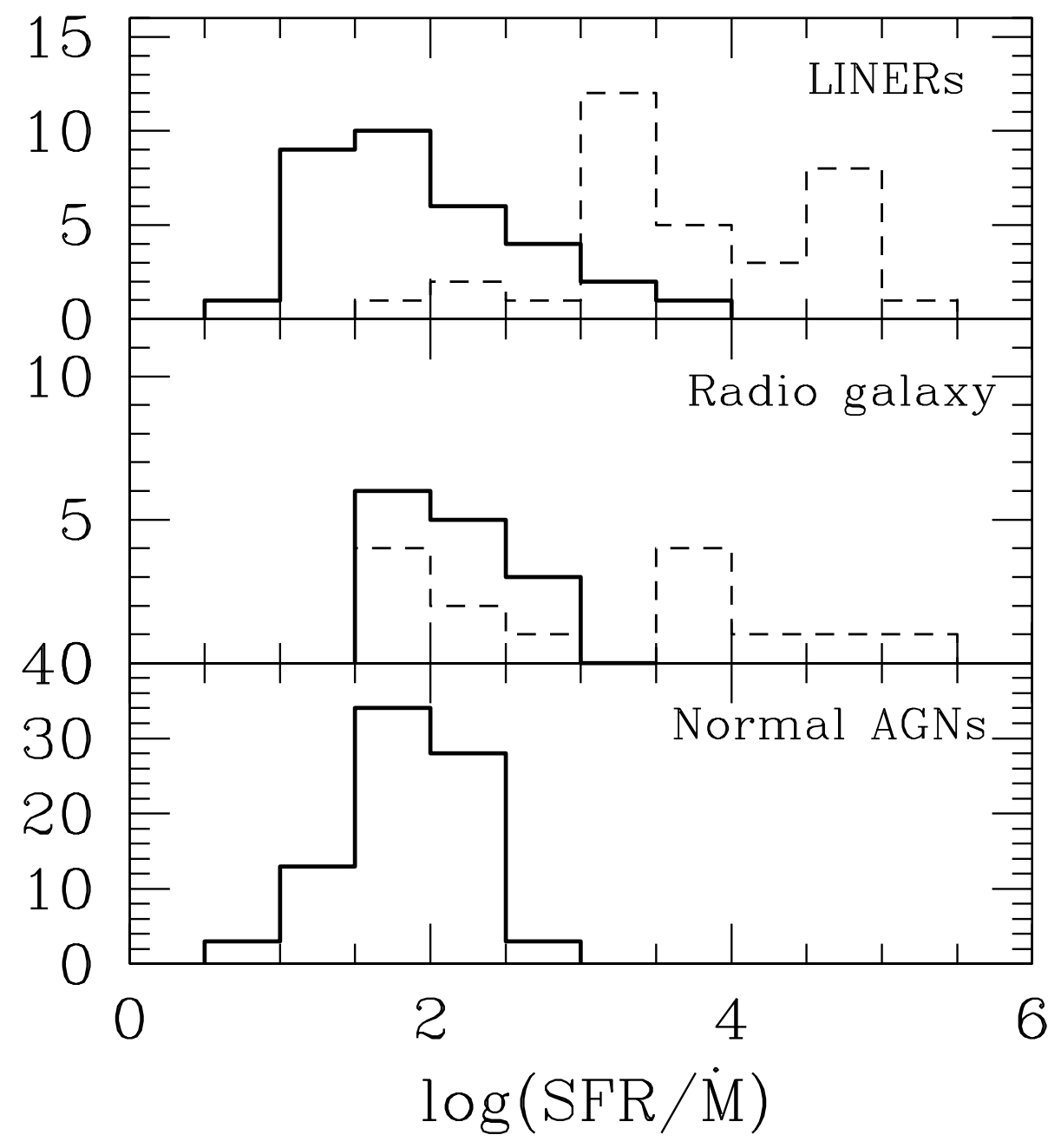

Fig. 2. - The histogram of SFR/ $\dot{M}$ for the sources in the sample: 33 LINERs (the upper panel), 14 radio galaxies (the middle panel), and 81 normal bright AGNs (52 Seyferts, 15 quasars and $14 \mathrm{NLS1s}$, the lower panel). The dashed lines in upper and middle panels are results of Satyapal et al. (2005), while solid lines are ours. 


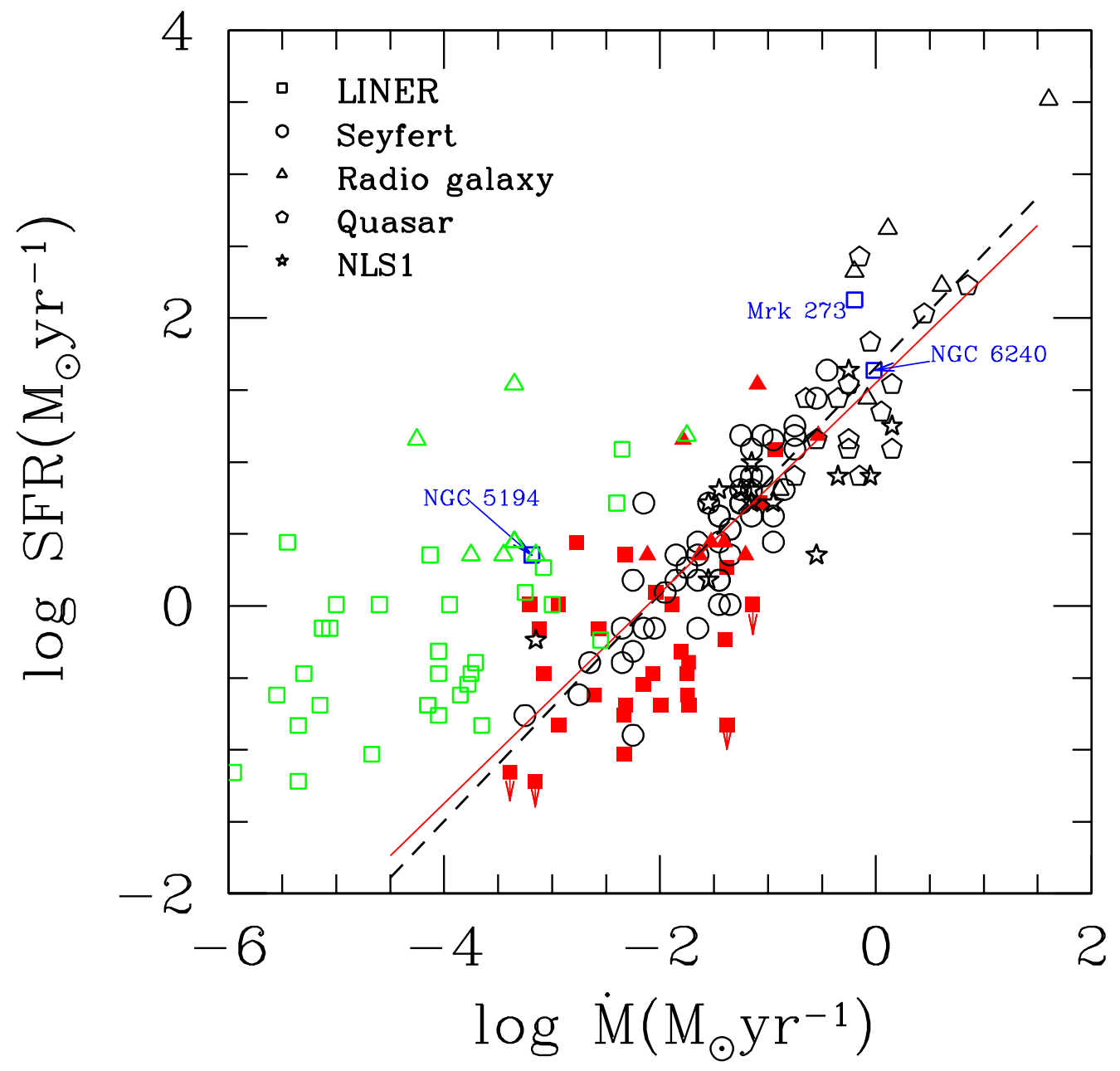

Fig. 3.- Relation between SFR and $\dot{M}$ for the whole sample. The green-open squares are results of Satyapal et al. (2005), while the red-solid squares are for the re-estimated accretion rates. The red-solid line represents the linear fit to the whole 128 sources, while black-dashed line is for the normal bright AGNs. 